\title{
Philosophiques
}

\section{Volition, ajustements moteurs et exploration des valeurs}

\section{Pierre Livet}

Volume 35, numéro 1, printemps 2008

Les valeurs de l'ironie (1) et le scepticisme à l'âge classique (2)

URI : https://id.erudit.org/iderudit/018240ar

DOI : https://doi.org/10.7202/018240ar

Aller au sommaire du numéro

Éditeur(s)

Société de philosophie du Québec

ISSN

0316-2923 (imprimé)

1492-1391 (numérique)

Découvrir la revue

Citer ce document

Livet, P. (2008). Volition, ajustements moteurs et exploration des valeurs.

Philosophiques, 35(1), 127-130. https://doi.org/10.7202/018240ar d'utilisation que vous pouvez consulter en ligne.

https://apropos.erudit.org/fr/usagers/politique-dutilisation/ 


\title{
Volition, ajustements moteurs et exploration des valeurs
}

\author{
PIERRE LIVET
}

Je dois d'abord signaler mon accord avec la stratégie générale de l'ouvrage de Joëlle Proust. Reformuler la notion de volition dans les termes du contrôle, la lier aux capacités d'ajustement et de révision de l'action, tenter d'étendre cette notion de contrôle et de révision à tous les niveaux de l'action, du niveau moteur au niveau de la transformation de soi en relation avec une image de soi, en passant par le niveau de la régulation d'une activité complexe en fonction de son adéquation avec un contexte dans lequel on dispose de repères pour savoir si l'on satisfait des règles, lier la constitution de l'identité personnelle à ces capacités de contrôle, repenser le débat sur la tension entre liberté et déterminisme en fonction de cette problématique nouvelle, ce sont là des lignes de programme auxquelles j'adhère, et qui sont brillamment exposées et développées dans La nature de la volonté.

Mes quelques perplexités portent sur la possibilité d'utiliser à tous les niveaux la référence à un modèle de contrôle qui me semble surtout pertinent au niveau moteur. Je ne pense pas que cela soit totalement satisfaisant. Mais comme nous savons que Joëlle Proust s'inspire de la théorie mathématique du contrôle développée par Aubin, et que cette théorie va bien au-delà d'une application possible au niveau moteur, cette limitation est peut-être apparente, et tient à ce que, dans la collection Folio, il était sans doute difficile de pouvoir présenter une théorie fort complexe comme celle d'Aubin et ensuite de montrer comment elle s'appliquait dans les différents niveaux de l'action.

Il me reste à argumenter mes perplexités. Au niveau moteur, nous rencontrons une situation privilégiée. D'une part, dans la plupart de nos mouvements, nous disposons d'un "modèle inverse ", qui nous dit comment en fonction d'un état final souhaité $\mathrm{n}$ transformer cet état pour parvenir à notre situation motrice présente, et donc quelles sont les opérations qu'il va falloir déclencher pour reparcourir en sens inverse le chemin qui va nous mener de la situation présente à l'état final souhaité. Nous disposons aussi de "simulations internes » ou «modèles internes » qui lient à la commande d'une séquence de mouvements l'anticipation des réafférences proprioceptives, voire perceptives qui devraient nous revenir en fonction de ces mouvements, et nous disposons de moyens de comparer ces réafférences anticipées et les réafférences effectives et de corriger les mouvements pour arriver à nos fins. Tout cela suppose une mise en branle en parallèle des commandes motrices, de l'enregistrement de ces commandes (efférence) et de la comparaison entre les réafférences anticipées et les réafférences observées. Mais la mise en place de ces modèles inverses, de ces modèles internes qui sont aussi des modèles anticipatifs, la mise en parallèle de leurs déterminations, et la détermination des capacités de correction, 
tout cela a exigé de très longs apprentissages, depuis notre naissance. Il n'est pas du tout évident que nous disposions de ces capacités à d'autres niveaux, qu'il s'agisse du contrôle qui tient compte des règles propres à une situation nouvelle, ou, davantage, de celui qui nous permet d'agir de manière cohérente avec nos valeurs et de mieux correspondre à notre image de nous-mêmes.

Avant de montrer en quoi les trois types de contrôle diffèrent, revenons sur l'interprétation que donne Joëlle Proust des expériences très intéressantes de Haggard et de son équipe. Il a montré qu'une activation cérébrale liée à une décision de répondre à la consigne précède une autre activation liée à la sélection d'un type de mouvement particulier, et que la conscience de la décision d'agir est concomitante non pas de la première activation, mais de la seconde. Il a aussi montré que dans une action volontaire, notre estimation de la localisation temporelle des effets de l'action se trouve décalée par rapport à sa véritable localisation et rapprochée de l'initiation de l'action, tandis que notre localisation temporelle du moment du vouloir agir est repoussée vers le futur, si bien que l'intervalle entre les deux est raccourci, alors que le contraire se produit dans le cas d'une action déclenchée par stimulation intra-crâniale par l'expérimentateur (en remplaçant le moment du vouloir-agir par le moment de la prise de conscience des mouvements involontaires).

Proust voit dans ces expériences une réfutation de la théorie interprétative de la volition. Selon cette théorie, la volition n'est qu'une illusion rétrospective, une construction de notre esprit qui situe dans le passé de l'action son initiation conçue sous forme de volition. L'argument de Proust semble être que s'il s'agissait d'une reconstruction liée à l'observation de nos mouvements et de leurs effets, nous ne devrions pas faire de différence de localisation temporelle entre l'action volontaire et l'action déclenchée expérimentalement. Cette différence s'explique en revanche si la volition est bien réelle, puisque alors nous unifions plus facilement une action volontaire avec ses effets que nous ne le faisons pour une action non volontaire. Mais pour que cet argument porte, il faudrait que la conscience d'être à l'origine de l'action puisse tenir une place comparable dans l'expérience de l'action volontaire et dans celle de l'action involontaire (pour qu'on puisse ne pas faire de différence). Or même dans une reconstruction rétrospective, nous pouvons fort bien tenir compte du fait que notre expérience phénoménale dans les deux cas n'est pas la même. La première expérience de Haggard montre bien que la conscience de la volition est liée non pas à une préparation globale, ou à une sorte de délibération implicite, mais plus particulièrement à la sélection d'un mouvement, donc à ce qui permet de lancer les anticipations de réafférences et la correction du mouvement en cas de besoin. La volition pourrait n'être qu'une reconstruction rétrospective qui dépend de ce processus de mise en branle d'un contrôle possible du mouvement. Quelle que soit d'ailleurs l'interprétation (volition reconstruction ou volition active), la conscience phénoménale de l'agir peut rester épiphénoménale et ne pas avoir d'incidence causale. La concomitance de cette conscience d'agir avec la sélection du mouvement pourrait faire de cette 
conscience une simple manifestation de la mise en route de ce qui permet le contrôle. La théorie de la volition comme contrôle peut toujours nous conduire à faire de la conscience de la volition un épiphénomène du contrôle. Les expériences en question ne semblent pas pouvoir trancher entre une interprétation où la volition a bien un rôle causal et celle où elle est un épiphénomène.

Dans le cas du contrôle "épisodique", selon les termes de Koechlin, nous ne disposons pas de ces ajustements quasi instantanés entre réafférences anticipées et réafférences observées. Ce contrôle consiste en effet à tenir compte des conditions induites par la situation, qu'il s'agisse de buts endogènes à l'agent, ou de règles propres à la situation du moment, où de l'influence de l'histoire de l'agent avant cette situation. Un tel ajustement immédiat ne serait possible que si nous avions déjà appris les valeurs et règles du contexte par le passé, et qu'il s'agisse donc du déclenchement de comportements routiniers. Mais si les modifications de contexte observées ne se sont pas souvent reproduites dans le passé et n'ont pas donné lieu à apprentissage, nous ne pouvons pas utiliser de tels ajustements. Ou bien nous disposons de représentations de situations prototypiques dont certains traits se retrouvent dans la nouvelle situation, et nous tentons des actions similaires à celles qui ont réussi dans le passé pour ces situations prototypiques. Ou bien nous savons seulement reconnaître certains traits saillants mais partiels de la situation. Ces traits activent en nous d'autres traits d'action à éviter ou à favoriser. Nous lançons alors seulement des actions qui peuvent éviter ces traits négatifs. Pour les traits positifs, nous tentons des actions, des constructions, et nous les modifions en fonction de l'estimation que nous faisons de leur proximité avec ces traits positifs. Ainsi un bricoleur qui tente de construire un mur tente de ne pas faire déborder latéralement sa brique par rapport aux briques déjà posées en-dessous. Le contrôle de l'action se fait donc avec des boucles rétroactives beaucoup plus longues, puisqu'il faut pouvoir observer les effets de l'action, parfois un temps après avoir fait le mouvement, pour pouvoir corriger. Nous améliorons nos performances en disposant de modèles mentaux des propriétés structurelles des objets que nous manipulons et des formes que nous devons obtenir.

Mais quand il s'agit de contrôler la manière dont nos états mentaux, nos désirs, préférences, sont bien en cohérence avec nos valeurs et avec l'image que nous souhaitons avoir, nous n'avons pas la plupart du temps de tels modèles mentaux — qui portent sur les formes des objets à produire — à consulter. Nous disposons bien de traits positifs ou négatifs, mais ils restent des qualités abstraites, et nous devons pouvoir découvrir leurs implications pour des conduites qui règlent nos interactions avec autrui. C'est toujours possible quand nous nous bornons à assurer des routines de comportement, qui se sont mises en place quand nous avons imité ceux qui nous paraissaient manifester ces valeurs. Mais si nous voulons satisfaire les valeurs que nous visons, nous sommes souvent dans des situations où nous ne disposons pas de modèles à imiter.

De plus, les valeurs que nous visons sont variées, et une même situation peut être évaluée selon des critères inspirés par des valeurs différentes, ce qui 
donne lieu à des évaluations contradictoires entres elles (telle conduite est supérieure à telle autre selon tel critère et telle valeur, mais inférieure selon telle autre). Il ne nous est pas possible de prétendre simplement mettre en cohérence nos actes et nos valeurs. Nous avons à explorer et découvrir ce que peuvent être les manières de résoudre ces problèmes d'incohérences entre évaluations. Nous pouvons tenter des révisions diverses, mais nous n'assurons par là que des remises en cohérence locales, et nous ne sommes pas assurés qu'une révision locale n'aura pas à plus long terme des conséquences pour d'autres valeurs qui introduiront des incohérences plus grandes.

Il faudrait donc apporter des nuances à l'approche de Joëlle Proust, en insistant davantage sur l'analyse des processus d'apprentissage et d'exploration qui permettent de nous donner des repères pour les corrections de nos actions et sur la diversité de ces processus. Ces processus ne sont pas équivalents selon les niveaux de contrôle qui sont en question. L'exploration des valeurs est bien plus incertaine que l'apprentissage des mouvements qui permettent d'atteindre un objet. Cela va d'abord dans le sens de la conception purement comparative de la liberté proposée par Joëlle Proust (nous ne sommes pas libres dans l'absolu, nous sommes plus libres si nous sommes capables de contrôles à plus de niveaux différents). Mais l'incertitude de l'exploration des valeurs nous laisse une forme de liberté plus sérieuse. Comme les valeurs à découvrir sont multiples et que leurs implications pour nos actions peuvent être en conflit, nous ne sommes plus là dans le champ du déterminisme. Nous pouvons choisir tel ou tel critère pour évaluer nos actions, mais aussi, à un niveau supérieur, pour poursuivre l'exploration des valeurs dans tel sens plutôt que tel autre. Assurément, si nous sommes enclins à développer telle exploration plutôt que telle autre, il est possible de prétendre expliquer ce penchant par des déterminismes. Mais comme les valeurs en question restent à explorer et découvrir, les catégories du déterminisme dont nous disposerons pour proposer ces explications seront toujours en retard sur nos explorations et seront plus grossières, ce qui nous donne une autre forme de liberté, certes relative elle aussi, mais cette fois relative à l'ouverture du champ des valeurs et non plus à une hiérarchie de modes de contrôle déjà déterminée. 